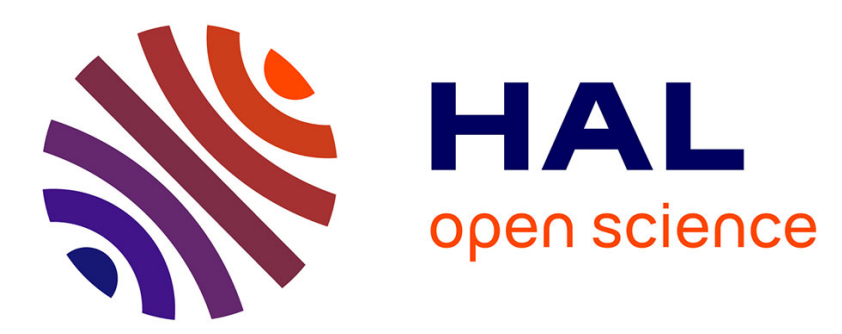

\title{
Excess wing and Johari-Goldstein relaxation in binary mixtures of glass formers
}

\author{
Daniele Prevosto, Khadra Kessairi, Simone Capaccioli, Mauro Lucchesi, \\ Pierangelo Rolla
}

\section{- To cite this version:}

Daniele Prevosto, Khadra Kessairi, Simone Capaccioli, Mauro Lucchesi, Pierangelo Rolla. Excess wing and Johari-Goldstein relaxation in binary mixtures of glass formers. Philosophical Magazine, 2007, 87 (3-5), pp.643-650. 10.1080/14786430600986111 . hal-00513771

\section{HAL Id: hal-00513771 \\ https://hal.science/hal-00513771}

Submitted on 1 Sep 2010

HAL is a multi-disciplinary open access archive for the deposit and dissemination of scientific research documents, whether they are published or not. The documents may come from teaching and research institutions in France or abroad, or from public or private research centers.
L'archive ouverte pluridisciplinaire HAL, est destinée au dépôt et à la diffusion de documents scientifiques de niveau recherche, publiés ou non, émanant des établissements d'enseignement et de recherche français ou étrangers, des laboratoires publics ou privés. 


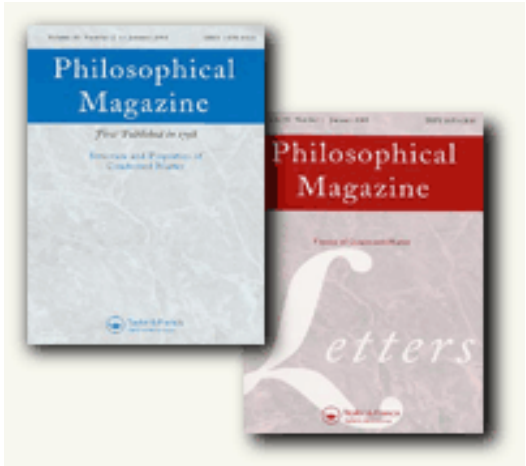

\section{Excess wing and Johari-Goldstein relaxation in binary mixtures of glass formers}

\begin{tabular}{|c|c|}
\hline Journal: & Philosophical Magazine \& Philosophical Magazine Letters \\
\hline Manuscript ID: & TPHM-06-May-0158.R2 \\
\hline Journal Selection: & Philosophical Magazine \\
\hline $\begin{array}{r}\text { Date Submitted by the } \\
\text { Author: }\end{array}$ & 22-Aug-2006 \\
\hline Complete List of Authors: & $\begin{array}{l}\text { Prevosto, Daniele; Department of Physics } \\
\text { Kessairi, Khadra; Department of Physics University of Pisa } \\
\text { Capaccioli, Simone; CNR-INFM Soft } \\
\text { Lucchesi, Mauro; Department of Physics University of Pisa } \\
\text { Rolla, Pierangelo; Department of Physics University of Pisa }\end{array}$ \\
\hline Keywords: & dielectric materials, disordered systems, glass transition \\
\hline Keywords (user supplied): & excess wing, Johari Goldstein relaxation, mixture \\
\hline
\end{tabular}

\section{s scholarONE" \\ Manuscript Central}




\title{
Excess wing and Johari-Goldstein relaxation in binary mixtures of glass formers
}

\author{
D. PREVOSTO*,1,2, K. KESSAIRI 1,2, S. CAPACCIOLI 2,3, M. LUCCHESI 1,2, P.A. ROLLA \\ 1,2 \\ 1 polyLab-CNR, Largo. B. Pontecorvo 3, 56127 Pisa, Italy \\ 2 Dipartimento di Fisica, Università di Pisa, Largo. B. Pontecorvo 3, 56127 Pisa, Italy \\ 3 CNR-INFM Soft, Piazzale A. Moro 2, 00185, Roma, Italy \\ *Corresponding author. Email: prevosto@df.unipi.it
}

\begin{abstract}
Dielectric loss spectra of pure quinaldine and tert-butylpyridine and their mixtures with tri-styrene are presented. The pure systems present an excess wing and no secondary peaks in the temperature interval from above to well below the glass transition. However, when mixed in low concentration with tri-styrene the excess wing is replaced by a distinct secondary peak. This distinct process can be identified as a Johari-Goldstein relaxation within the Coupling Model interpretation. In the frame of the Coupling Model the transition from the relaxation scenario with the excess wing to that with a distinct secondary peak is related to the increase of intermolecular constraints. In our case this increase of constraints is due to the low mobility component of the mixture (tri-styrene).
\end{abstract}

Keywords: Glass formers; mixture; excess wing; Johari Goldstein relaxation; nearly constant loss

\section{AMS Subject Classification:}

\section{Introduction}

The hypothesis of a two step relaxation dynamics (for example see Ref. [1-3] and references therein), and the recent investigations about the origin of secondary relaxation in for glass formers [4-12] raised the question whether the secondary process is an universal feature of the relaxation dynamics. Experimentally, more than one secondary process can be observed in some glass formers with complex molecular structure $[6,9,10]$. Two main mechanisms for the secondary processes are generally proposed: an intramolecular motion of some molecular subgroup dynamically decoupled from the rest of the molecule [13], or a small angle motion of the entire molecule as a whole [3,5]. The process originating from the last mechanism is usually referred as Johari Goldstein (JG) process, and it is supposed to reflect an universal relaxation mechanism. However, loss spectra of some glass formers show no evidence of any secondary peak, but only an extra loss contribution (excess wing, EW) in the high frequency tail of the structural process. Such a contribution appears as a power law with exponent smaller than that characterizing the high frequency part of the structural peak. The microscopic origin of the EW was debated and it was discussed whether it is a secondary peak submerged by the structural or it is a relaxation feature connected to the structural process [16-27]. In some cases the appearance of the EW is only a matter of a reduced frequency or temperature interval of investigation: by extending them it is observed that the EW becomes a secondary peak separated from the structural one $[14,15,21]$. However, these are particular cases 
that cannot be confused with those where the EW is detected even measuring over a wide frequency interval in the deep glassy state.

Some articles discussed the origin of the EW proposing different interpretations. Early approaches interpreted the EW in the frame of the mode coupling theory, but without success [16], or, on the basis of scaling arguments, as an inherent part of the $\alpha$-relaxation [17,18]. A new approach to the EW in term of the MCT was recently proposed [19] but not verified on the available data. Kudlik and co-authors [20] suggested that supercooled liquids can be classified in two classes, depending if in the glassy state they show to an EW or a $\beta$-relaxation mechanism. Later on, different experimental results have cast doubts on the validity of this classification [22,23]. Schneider and coworkers observed the EW of glycerol and propylene carbonate transforming into a secondary peak after aging at temperatures below $\mathrm{T}_{\mathrm{g}}[26]$. They explained such behaviour suggesting that different dependence on aging of the structural and secondary peaks favours the observation of the latter. Similarly, dielectric spectra of the trimer of propylene glycol at high pressure showed an EW that, after aging below $\mathrm{T}_{\mathrm{g}}$, transformed into a shoulder [25]. Dielectric measurements on a family of polyalcohols and polymers showed that the secondary relaxation peak gets close to the structural with decreasing the number of repeating units, until it merges forming an EW [12,27,28]. The application of very high pressure transform the EW of threithol and glycerol into a partially merged secondary peak [27]. Finally, in three cases the EW was changed into a secondary peak after mixing a polar glass former with one having a negligible dielectric signal (picoline in o-terphenyl, picoline in tri-styrene, and propylene carbonate in poly(methyl mathacrylate)) [22-24].

In the case of polyalcohols and oligomers the appearance of a secondary peak at large number of repeating units was explained in terms of increase of the effects of the many molecules dynamics and intermolecular coupling [4]. This explanation was quantitatively inspired by the Coupling Model (CM) [3] predicting that the frequency separation of structural and secondary JohariGoldstein, JG, peaks is strictly related to the coupling parameter (n),

$n=\left[\log \left(\tau_{\alpha, \max }\right)-\log \left(\tau_{J G}\right)\right] /\left[\log \left(\tau_{\alpha, \max }\right)+11.7\right]$

where $\tau_{\alpha, \max }=1 /\left(2 \pi v_{\alpha, \max }\right)$ and $\tau_{\mathrm{JG} \text {,max }}=1 /\left(2 \pi \nu_{\mathrm{JG}}\right)$ are the relaxation times corresponding to the maximum frequency $v_{\alpha, \max }$ and $v_{\mathrm{JG}}$ of the structural and JG peaks, respectively. The coupling parameter, which is related to the stretching of the Kohlrausch Williams Watts (KWW) function used to fit the structural peak, $n=1-\beta_{\mathrm{KWW}}$, is usually considered a measure of the intermolecular coupling. The greater is the value of $\mathrm{n}$ the greater is the effect of the intermolecular constraints leading to the many molecules dynamics. According to Eq. (1) for a fixed value of $\tau_{\alpha, \max }$ (or $v_{\alpha, \max }$ ) the separation of the JG peak must be larger for greater values of $n$. In both polyalcohols and oligomers, for larger number of repeating units a larger value of $n$ was observed and accordingly a secondary peak separated from the structural [4,12,28-29]. Instead, for short oligomers a smaller value of $\mathrm{n}$ was found and the secondary peak was replaced by an EW. The same explanation was recently [30] successfully used to interpret the appearance of a secondary peak in mixtures of oterphenyl in tri-styrene and picoline in tri-styrene. Consistently the scenario can be explained considering that the EW is a secondary peak of the JG type [4], which for small enough values of $\mathrm{n}$ is so close to the structural peak that it results submerged by it.

In this work we extended previous investigations considering the secondary dynamics of two pure systems, presenting excess wing, and their mixtures with a non-polar compound,. In this latter case the EW was replaced by a secondary peak. We evidenced the role of intermolecular interactions in defining the two relaxation scenario and we interpreted the emerged secondary peak as a JG relaxation in term of the $\mathrm{CM}$.

\section{Experiment}




\section{Results and Discussion}

Dielectric loss spectra (Fig. 1) of TBP and Qn above $T_{g}$ show the conductivity contribution, due to the ionic impurity, appearing as a rise of the signal at low frequencies (for clarity the contribution is shown only for few spectra in Fig. 1), and the contribution due the structural relaxation dynamics, appearing as a peak. These two processes slow down with temperature, and finally below $\mathrm{T}_{\mathrm{g}}$ also the structural peak disappears from the investigated frequency window. On the high frequency we observe an excess loss contribution, appearing above $\mathrm{T}_{\mathrm{g}}$ as a deviation from the one side Fourier Transform of the KWW function fitting the structural peak (see continuous lines in Fig.1). In Fig. 1 the power law describing the excess wing is evidenced in the loss spectra of TBP and Qn measured at $169 \mathrm{~K}$ and $188 \mathrm{~K}$, respectively. The exponents of the power laws are $-0.29 \pm 0.01$ and $-0.40 \pm 0.01$, respectively, both smaller than those characterising the high frequency tail of the structural peak as determined from the KWW fit. This difference make it possible to distinguish between the excess wing and the common power law characterizing the high frequency tail of the structural peak.

The excess wing in both samples moves towards lower frequency with the structural peak and disappears from the investigated frequency window well below $\mathrm{T}_{\mathrm{g}}$ (Fig. 1). In this region the loss spectra evidence a weak decrease with frequency; such loss contribution is well studied in ionic conductors and it is usually called nearly constant loss [32,33]. This behaviour can be reasonably fitted by a second power law whose exponent decreases with temperature (Fig. 1). For Qn the exponent decreases from $-0.21 \pm 0.01$ at $143 \mathrm{~K}$ to $0.15 \pm 0.01$ at $113 \mathrm{~K}$, and in TBP from $0.12 \pm 0.01$ at $163 \mathrm{~K}$ to $0.11 \pm 0.01$ at $133 \mathrm{~K}$. It is worth to note that a similar phenomenology was reported in Ref. [3] for few other glass forming systems, namely propylene carbonate, glycerol, cresolphthaleindimethyl ether and N-methyl- $\varepsilon$-caplolactam. Our results on the pure TBP and Qn confirm and extend those reported in previous works in two points. First of all, we confirm that the excess wing is a relaxation feature characteristic of systems with a narrow structural peak. The coupling parameter, which is a measure of the deviation of the structural peak from the Debye relaxation, is $0.30 \pm 0.03$ for Qn and $0.37 \pm 0.02$ for TBP (Fig. 2), which compares with $\mathrm{n}=0.29$ of Glycerol and other systems showing EW, as it was already discussed in other publications, for example $[4,30]$. The second important result is that in system with EW below $\mathrm{T}_{\mathrm{g}}$ it is possible to observe a weak 
frequency dependence of dielectric loss, which cannot ascribed to the extrapolation of the high frequency tail of the structural peak or to the extrapolation of the EW.

The main goal of our work is the investigation of the relaxation mechanism at the basis of the EW. To extend the results of previous papers $[12,23,24,30]$, we investigated the possibility to transform the EW into a secondary peak by changing intermolecular coupling among the investigated molecules. In our work we changed intermolecular coupling by mixing the polar TBP and Qn with 3Styr. The dipole moment of the former molecules is more than a factor 10 greater than that of 3 Styr, so that we can expect the a ratio of more than 2 orders of magnitude between the dielectric strength of the relaxations. The difference in the intensity of the signals assures that dielectric response of the mixture reflects the relaxation dynamics of TBP and Qn molecules dispersed in the 3Styr matrix, and no direct contribution of 3Styr molecules is observed.

Dielectric spectra of pure Qn and TBP present a narrower structural relaxation compared to that of their mixtures with 3Styr (Fig. 2). The coupling parameter changes from $n=0.37$ to 0.55 (from pure Qn to the mixture with Qn weight ratio of $5 \%$ ) and from $n=0.30$ to 0.58 (from pure TBP to the mixture with TBP weight ratio of $16 \%$ ). It is common that the structural peak in mixtures with similar percentages of the two components is broader than those of the pure systems. In such cases the broadening is attributed to the effect of local composition heterogeneities, usually named as concentration fluctuations [34]. Instead, in the limit of high dilution (concentration less than 1\%) the broadness of the observed structural peak can vary between that of the pure host system, and that of a Debye function [35] mainly depending on the relative mobility of the molecules. In the case of low, but not dilute, concentration of a polar component with an apolar one characterized by lower mobility, as in our mixtures, the concentration fluctuations contribution should be negligible, but a certain broadening is observed. The origin of the latter was ascribed to the larger effects of dynamic constraints imposed to the polar molecules from the slower molecules of the apolar component. This effect was related to the separation between structural and JG secondary peak [30]. The CM predicts that the frequency separation between the JG secondary and the structural peaks is larger for systems with greater dynamic heterogeneity, i.e. larger value of coupling parameter (Eq. 1). In a neat system a good estimation of dynamic heterogeneity is the value of $n$ determined from the broadness of the structural peak. Usually in mixtures such estimation is not reliable especially when concentration fluctuations is important, whose effects lead to a broadening of the structural peak reflecting only statistic and not dynamic effects. A discussion of this topic together with a detailed presentation of the extension of the CM to bi-component mixtures is in Ref. [30]. In our case fluctuations concentration can be neglected due to the low concentration of the polar component: actually one polar molecule is surrounded mainly by styrene repeating units and a concentration fluctuations cannot significantly change this environment. Our data confirm that a broadening of the structural peak, due to an increase of many molecules interactions, leads to a greater separation between secondary JG relaxation and structural peak, as discussed in [30]. In fact, in both pure systems, with narrow structural peak, only the EW is present, whereas in the mixtures, presenting a broader structural peak, a well separated secondary peak appears. The new peak is sensitive to temperature and the activation energy estimated below $T_{g}$ is $54 \pm 1 \mathrm{~kJ} / \mathrm{mol}$ for the Qn mixture and $29 \pm 1 \mathrm{~kJ} / \mathrm{mol}$ for the TBP mixture.

The interpretation here proposed consider the EW as a hidden secondary relaxation. Such hypothesis can be quantitatively confirmed in term of the CM. In fact, Eq. (1) can be rewritten as $v_{\mathrm{JG}}=\left(2 \pi \mathrm{t}_{\mathrm{c}}\right)^{-\mathrm{n}} v_{\alpha}{ }^{(1-n)}$, where $\mathrm{t}_{\mathrm{c}}=2 \mathrm{ps}$, which allows to calculate the position of the JG relaxation once the values of $v_{\alpha}$ and $n$ are known [3,4]. The calculated values of $v_{\mathrm{JG}}$ are in fair agreement with those of the observed secondary peaks in both mixtures (vertical arrows in Fig. (2)). In the case of the pure system the JG relaxation is predicted in the region of the EW, hidden beneath the structural peak. The CM suggests as relaxation mechanism a continuous evolution from a local restricted relaxation process of the whole molecules $(\mathrm{JG})$ to a cooperative relaxation (structural process) [3,4]. Both the excess wing and the secondary process emerging in the neat system and mixture, respectively, reflect the restricted and non cooperative motion of the whole molecule. 


\section{Conclusion}

Dielectric loss spectra of pure quinaldine and tert-butylpyridine and their mixtures with tri-styrene are presented. The pure systems present an excess wing and no secondary peak, whereas the mixtures with tri-styrene show a distinct secondary peak. In the frame of the Coupling Model the transition from the relaxation scenario with excess wing to that with secondary peak is related to the increase of intermolecular constraints induced by the low mobility component of the mixture (tristyrene). The secondary peak observed in the mixture can be interpreted as Johari-Goldstein.

\section{References}

[1] W. Götze, M. Sperl Phys. Rev. Lett. 92, 105701 (2004).

[2] H. Tanaka J. Non-Cryst. Solids 351, 3396 (2005); H. Tanaka J. Non-Cryst. Solids 351, 3385 (2005).

[3] K.L. Ngai, M. Paluch J. Phys. Chem. B, 107, 6865 (2003); K.L. Ngai J. Phys.: Condens. Matter 15, S1107 (2003).

[4] K.L. Ngai, M. Paluch J. Chem. Phys. 120, 857 (2004).

[5] M. Vogel, C. Tschirwitz, G. Schneider, C. Koplin, P. Medick, E. Rössler J. Non-Cryst. Solids 307-310, 326 (2002).

[6] D. Prevosto, S. Capaccioli, M. Lucchesi, P.A. Rolla and K.L. Ngai J. Chem. Phys. 120, 4808 (2004).

[7] N.B. Olsen, T. Christensen, J.C. Dyre, Phys. Rev. E 62, 4435 (2000).

[8] H. Wagner, R. Richert, J. Phys. Chem. B 103, 4071 (1999).

[9] M. Paluch, S. Pawlus, S. Hensel-Bielowka, E. Kaminska, D. Prevosto, S. Capaccioli, P. A. Rolla, K.L. Ngai, J. Chem. Phys. 122, 234506 (2005).

[10] D. Pisignano, S. Capaccioli, R. Casalini, M. Lucchesi, P.A. Rolla, A. Justl, E. Rössler J. Phys.: Condens. Matter 13, 4405 (2001).

[11] S. Hensel-Bielowka, M. Paluch, Phys. Rev. Lett., 89, 025704 (2002).

[12] J. Mattsson, R. Bergman, P. Jacobsson, L. Börjesson Phys. Rev. Lett., 90, 075702 (2003).

[13] N.G. McCrum, B.E. Read, and G. Williams, Anelastic and Dielectric Effects in Polymeric Solids (Wiley, New York, 1967).

[14] M.T. Viciosa, C.M. Rodriguez, M. Dionisio J. Non-Cryst. Solids 351, 14 (2005).

[15] S. Maslanka, M. Paluch, W.W. Sulkowski, C.M. Roland J. Chem. Phys. 122, 084511 (2005)

[16] A. Hoffmann, F. Kremer, E. W. Fischer, and A. Schönhals, in Disorder Effects on Relaxational Processes, edited by R. Richert and A. Blumen (Springer, Berlin, 1994), p. 309.

[17] P. K. Dixon, L. Wu, S. R. Nagel, B. D. Williams, and J. P. Carini, Phys. Rev. Lett. 65, 1108 (1990).

[18] R. V. Chamberlin, Phys. Rev. B 48, 15638 (1993).

[19] H.Z. Cummins J. Phys.: Condens. Matter 17, 1457 (2005).

[20] A. Kudlik, S. Benkhof, T. Blochowicz, T. Tschirwitz, E.A. Rössler J. Mol. Struct. 479, 201 (1999).

[21]S. Capaccioli, D. Prevosto, M. Lucchesi, P.A. Rolla, R. Casalini, K.L. Ngai J. Non-Cryst. Solids 351, 2643 (2005).

[22] T. Blochowicz, E.A. Rössler, Phys. Rev. Lett. 92, 225701 (2004).

[23] T. Blochowicz, Broadband Dielectric Spectroscopy in Neat and Binary Molecular Glass

Formers; ISBN 3-8325-0320-X, Logos Verlag:Berlin, 2003.

[24] C. Svamberg, R. Bergman, P. Jacobsson Europhys. Lett. 64, 358 (2003).

[25] R. Casalini, C. M. Roland, Phys. Rev. Lett. 91, 015702 (2003).

[26] U. Schneider, R. Brand, P. Lunkenheimer, A. Loidl Phys. Rev. Lett. 84, 5560 (2000).

[27] S. Hensel-Bielowka, S. Pawlus, C.M. Roland,J. Ziolo, M. Paluch Phys. Rev. E 69, 050501 (2004). 
[28] C. Leon, K.L. Ngai, C.M. Roland J. Chem. Phys., 110, 11585 (1999).

[29] A. Doss, M. Paluch, H. Sillescu, G. Hinze Phys. Rev. Lett., 88, 095701 (2002).

[30] S. Capaccioli, K.L. Ngai J. Phys. Chem. B, 109, 9727 (2005).

[31] K. Kessairi, S. Capaccioli, et al.in preparation.

[32] K.L. Ngai, C. Leon Phys. Rev. B 66, 064308 (2002).

[33] R. Casalini, K.L. Ngai J. Non-Cryst. Solids 293-295, 318 (2001).

[34] R. Kant, S. K. Kumar, Macromolecules 36, 10087 (2003).

[35] W. Huang, R. Richert J. Chem Phys. 124, 164510 (2005).

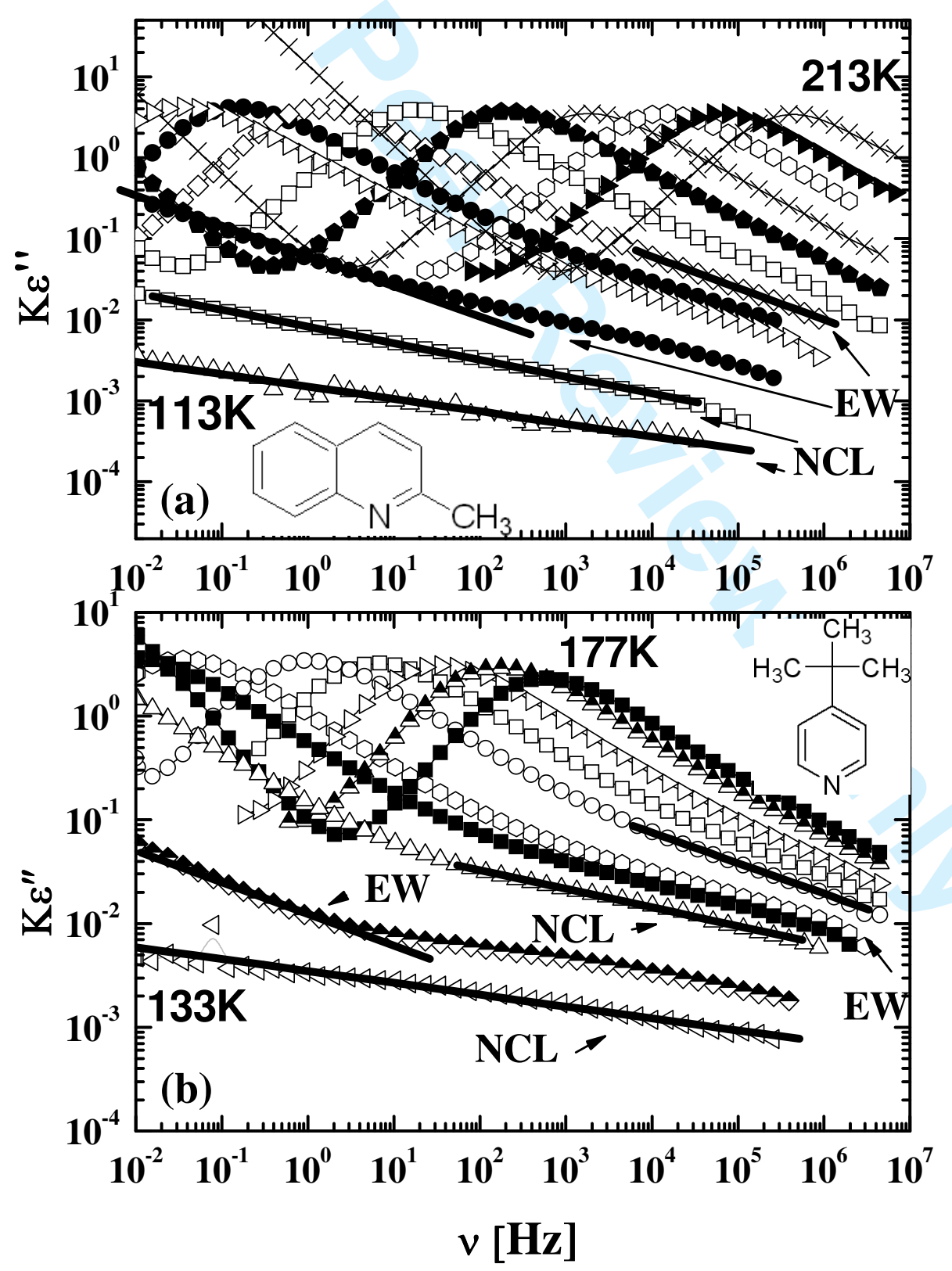


Fig.1. (a) Double logarithmic representation of dielectric loss spectra of Quinaldine at the temperatures (from the right to the left) 213, 208, 204, 200, 196, 192, 188, 185, 183, 173, 143, 113 $\mathrm{K}$. The EW contribution is evidenced in the spectra at 188 and $143 \mathrm{~K}$, whereas the NCL is evidenced in the spectra at 173 and $113 \mathrm{~K}$. In the inset the molecular structure is reported. (b) Double logarithmic representation of dielectric loss spectra of Tert-butylpyridine at the temperature (from the right to the left) 177, 175, 173, 171, 169, 166, 165, 163, 158, $133 \mathrm{~K}$. The EW contribution is evidenced in the spectra at 169 and $158 \mathrm{~K}$, whereas the NCL is evidenced in the spectra at 163 and $133 \mathrm{~K}$. In the inset the molecular structure is reported.

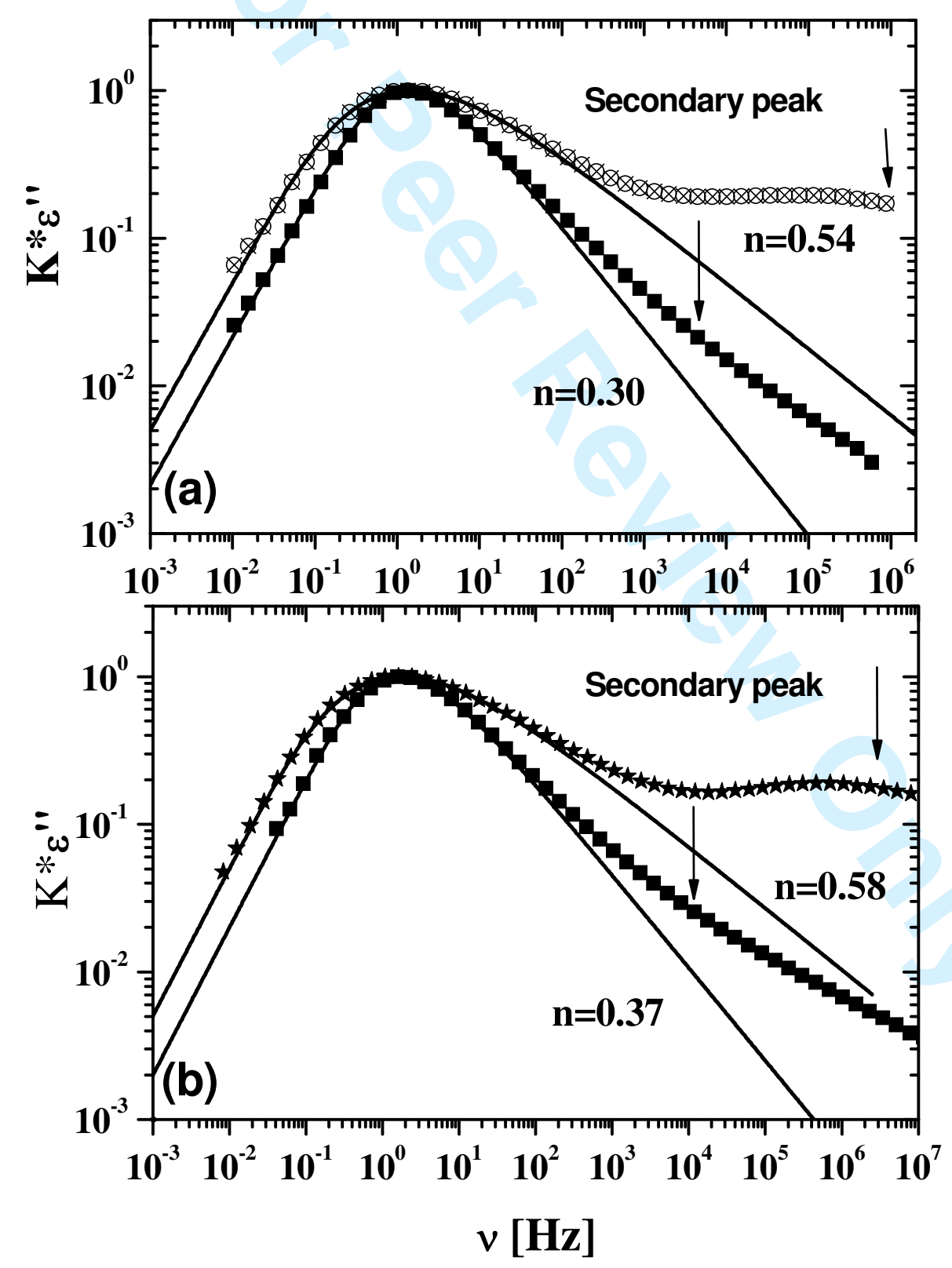

Fig. 2 Normalized loss spectra of pure (a) Qn (squares, $188 \mathrm{~K}$ ) and mixture of $5 \%$ Qn in 3Styr (crossed open circles, $236 \mathrm{~K}$ ) and (b) pure TBP (squares, $169 \mathrm{~K}$ ) and mixture of $16 \% \mathrm{TBP}$ in 3Styr (stars, $223 \mathrm{~K}$ ). Each couple of spectra were measured so that the frequency of structural peak is 
nearly the same. When necessary a small horizontal shift factor was applied to obtain an exact match of the maxima. In both panels the continuous curves represent KWW curves to fit the structural peak, with reported values of the coupling parameters, $n=1-\beta_{\mathrm{KWw}}$. The vertical arrows indicate the position of the JG secondary relaxation predicted by the coupling model. 